\title{
Correct expression of the material derivative in continuum physics
}

\section{Bohua Sun $^{1}$}

1 School of Civil Engineering 8 Institute of Mechanics and Technology Xi'an University of Architecture and Technology, Xi'an 710055, China http://imt.xauat.edu.cn

email: sunbohua@xauat.edu.cn

(Dated: April 27, 2020)

\begin{abstract}
The material derivative is important in continuum physics. This Letter shows that the expression $\frac{d}{d t}=\frac{\partial}{\partial t}+(\boldsymbol{v} \cdot \boldsymbol{\nabla})$, used in most literature and textbooks, is incorrect. The correct expression $\frac{d(:)}{d t}=\frac{\partial}{\partial t}(:)+\boldsymbol{v} \cdot[\nabla(:)]$ is formulated. The solution existence condition of Navier-Stokes equation has been proposed from its form-solution, the conclusion is that "The Navier-Stokes equation has solution if and only if the determinant of flow velocity gradient is not zero, namely $\operatorname{det}(\boldsymbol{\nabla} \boldsymbol{v}) \neq 0 . "$
\end{abstract}

the material derivative with respect to time must be defined. For mass density $\rho(\boldsymbol{x}, t)$, flow velocity $\boldsymbol{v}(\boldsymbol{x}, t)=$ $v_{k} \boldsymbol{e}_{k}$, and stress tensor $\boldsymbol{\sigma}=\sigma_{i j} \boldsymbol{e}_{i} \otimes \boldsymbol{e}_{j}$, the material derivatives are given by:

$$
\begin{gathered}
\frac{d \rho}{d t}=\frac{\partial \rho}{\partial t}+\frac{\partial \rho}{\partial x_{k}} \frac{\partial x_{k}}{\partial t}=\frac{\partial \rho}{\partial t}+v_{1} \frac{\partial \rho}{\partial x^{1}}+v_{2} \frac{\partial \rho}{\partial x^{2}}+v_{3} \frac{\partial \rho}{\partial x^{3}},(1) \\
\frac{d \boldsymbol{v}}{d t}=\frac{\partial \boldsymbol{v}}{\partial t}+\frac{\partial \boldsymbol{v}}{\partial x_{k}} \frac{\partial x_{k}}{\partial t}=\frac{\partial \boldsymbol{v}}{\partial t}+v_{1} \frac{\partial \boldsymbol{v}}{\partial x^{1}}+v_{2} \frac{\partial \boldsymbol{v}}{\partial x^{2}}+v_{3} \frac{\partial \boldsymbol{v}}{\partial x^{3}}
\end{gathered}
$$

and

$$
\frac{d \boldsymbol{\sigma}}{d t}=\frac{\partial \boldsymbol{\sigma}}{\partial t}+\frac{\partial \boldsymbol{\sigma}}{\partial x_{k}} \frac{\partial x_{k}}{\partial t}=\frac{\partial \boldsymbol{\sigma}}{\partial t}+v_{1} \frac{\partial \boldsymbol{\sigma}}{\partial x^{1}}+v_{2} \frac{\partial \boldsymbol{\sigma}}{\partial x^{2}}+v_{3} \frac{\partial \boldsymbol{\sigma}}{\partial x^{3}}
$$

respectively, where $t$ is time, $\boldsymbol{x}=x_{k} \boldsymbol{e}_{k}$ is position, $\boldsymbol{e}_{k}$ is a base vector and $v_{k}=\frac{\partial x_{k}}{\partial t}$ is the flow velocity.

In most textbooks, handbooks and encyclopedia, such as Refs. 1-12, the above material derivatives are expressed as:

$$
\begin{gathered}
\frac{d \rho}{d t}=\frac{\partial \rho}{\partial t}+(\boldsymbol{v} \cdot \nabla) \rho, \\
\frac{d \boldsymbol{v}}{d t}=\frac{\partial \boldsymbol{v}}{\partial t}+(\boldsymbol{v} \cdot \nabla) \boldsymbol{v}
\end{gathered}
$$

and

$$
\frac{d \boldsymbol{\sigma}}{d t}=\frac{\partial \boldsymbol{\sigma}}{\partial t}+(\boldsymbol{v} \cdot \boldsymbol{\nabla}) \boldsymbol{v}
$$

where the gradient operator $\boldsymbol{\nabla}=\boldsymbol{e}_{k} \frac{\partial}{\partial x_{k}}$.

To simplify Eqs. (1)-(3) further, a differential operator is introduced as follows:

$$
\frac{d}{d t}=\frac{\partial}{\partial t}+(\boldsymbol{v} \cdot \nabla)
$$

This operator is used by most fluid mechanics textbooks, including well-known graduate textbooks such as Landau \& Lifshitz,[1, 2] Prandtl,[3, 4] Anderson,[5] Pope,[6] Cengel \& Cimbala,[7], Kundu et al.[8], Woan [9], wikipedia $[10,11]$ and britannica [12].
In the latest version of Landau \& Lifshitz,[13] the material derivative of flow velocity is given in another form:

$$
\frac{d \boldsymbol{v}}{d t}=\frac{\partial \boldsymbol{v}}{\partial t}+(\boldsymbol{v} \nabla) \boldsymbol{v}
$$

Besides the books mentioned above, there is a large amount of literature in fluid mechanics taking the same expressions as Refs. 1 and 13. This makes it difficult for readers to identify which expression for the material derivative is correct, causing great confusion to both students and scholars.

Although some authors, such as Lighthill,[14] Batchelor,[15] Frisch,[16], Nhan \& Nam [17], Xie,[18] and Zhao [19] have used the correct expression, it seems that most fluid mechanics textbooks and academic literature have adopted an incorrect expression for the material derivative as in Ref. 1 . Therefore, to revive the great influence of Landau in physics and fluid mechanics, we attempt to address this issue in this dedicated paper, where we revisit the material derivative to show why Eqs. (7) and (8) are incorrect, and derive a correct expression using standard tensor calculus.

In Landau \& Lifshitz, [1] the material derivative is given as:

$$
d x \frac{\partial \boldsymbol{v}}{\partial x}+d y \frac{\partial \boldsymbol{v}}{\partial y}+d z \frac{\partial \boldsymbol{v}}{\partial z}=(d \boldsymbol{r} \cdot \operatorname{grad}) \boldsymbol{v}
$$

Although Landau's fluid mechanics is well known worldwide, the above expression is incorrect.

\section{Proof:}

Since $\boldsymbol{v} \cdot \operatorname{grad}=\boldsymbol{v} \cdot \boldsymbol{\nabla}=\left(v_{i} \boldsymbol{e}_{i}\right) \cdot\left(\partial_{j} \boldsymbol{e}_{j}\right)=$ $v_{i, j} \boldsymbol{e}_{i} \cdot \boldsymbol{e}_{j}=v_{i, j} \delta_{i j}=\operatorname{div} \boldsymbol{v}$, and $(\boldsymbol{v} \cdot \operatorname{grad}) \boldsymbol{v}=$ $(\boldsymbol{v} \cdot \boldsymbol{\nabla}) \boldsymbol{v}=\left(v_{j, i} \boldsymbol{e}_{i} \cdot \boldsymbol{e}_{j}\right)\left(v_{k} \boldsymbol{e}_{k}\right)=\delta_{i j} v_{j, i} v_{k} \boldsymbol{e}_{k}=$ $v_{i, i} v_{k} \boldsymbol{e}_{k}=(\operatorname{div} \boldsymbol{v}) \boldsymbol{v}$, where the divergence is $\operatorname{div} \boldsymbol{v}=\frac{\partial v_{1}}{\partial x^{1}}+\frac{\partial v_{2}}{\partial x^{2}}+\frac{\partial v_{3}}{\partial x^{3}}$, so $(\operatorname{div} \boldsymbol{v}) \boldsymbol{v}=\left(\frac{\partial v_{1}}{\partial x^{1}}+\right.$ $\left.\frac{\partial v_{2}}{\partial x^{2}}+\frac{\partial v_{3}}{\partial x^{3}}\right) \boldsymbol{v}$. Thus:

$$
(\boldsymbol{v} \cdot \operatorname{grad}) \boldsymbol{v} \neq v_{1} \frac{\partial \boldsymbol{v}}{\partial x^{1}}+v_{2} \frac{\partial \boldsymbol{v}}{\partial x^{2}}+v_{3} \frac{\partial \boldsymbol{v}}{\partial x^{3}} .
$$


In the latest version of Landau \& Lifshitz, [13] for reasons which are unclear, the material derivative expression has been changed to $\frac{d \boldsymbol{v}}{d t}=\frac{\partial \boldsymbol{v}}{\partial t}+(\boldsymbol{v} \nabla) \boldsymbol{v}$; this is also incorrect.

\section{Proof:}

Since $\boldsymbol{v} \boldsymbol{\nabla}=\left(v_{i} \boldsymbol{e}_{i}\right)\left(\partial_{j} \boldsymbol{e}_{j}\right)=v_{i, j} \boldsymbol{e}_{i} \boldsymbol{e}_{j}$,

$(\boldsymbol{v} \nabla) \boldsymbol{v}=\left(v_{j, i} \boldsymbol{e}_{i} \boldsymbol{e}_{j}\right)\left(v_{k} \boldsymbol{e}_{k}\right)=\left(v_{j, i} \boldsymbol{e}_{i} \boldsymbol{e}_{j}\right)\left(v_{k} \boldsymbol{e}_{k}\right)$,

which indicates that $\boldsymbol{v} \boldsymbol{\nabla}$ is a 3 rd-order tensor rather than a vector. Thus:

$$
(\boldsymbol{v} \nabla) \boldsymbol{v} \neq v_{1} \frac{\partial \boldsymbol{v}}{\partial x^{1}}+v_{2} \frac{\partial \boldsymbol{v}}{\partial x^{2}}+v_{3} \frac{\partial \boldsymbol{v}}{\partial x^{3}}
$$

To obtain the correct formulation of the material derivative, we perform some basic tensor calculus [20]. The del operator is a vector differential operator, and defined by $\boldsymbol{\nabla}=\boldsymbol{e}_{i} \frac{\partial}{\partial x_{i}}$. An important note concerning the del operator $\boldsymbol{\nabla}$ is in order. Two types of gradients are used in continuum physics: left and right gradients. The left gradient is the usual gradient and the right gradient is the transpose of the forward gradient operator.

To see the difference between the left and right of gradients, consider a vector function $\boldsymbol{A}=A_{i}(\boldsymbol{x}) \boldsymbol{e}_{i}$. The left gradient of a vector $\boldsymbol{A}$ is $\boldsymbol{\nabla} \otimes \boldsymbol{A} \equiv \boldsymbol{e}_{j} \frac{\partial}{\partial x_{j}} \otimes$ $\left(A_{i} \boldsymbol{e}_{i}\right)=\frac{\partial A_{i}}{\partial x_{j}} \boldsymbol{e}_{j} \otimes \boldsymbol{e}_{i}=A_{i, j} \boldsymbol{e}_{j} \otimes \boldsymbol{e}_{i}$, or written as $\boldsymbol{\nabla} \boldsymbol{A} \equiv$ $\boldsymbol{e}_{j} \frac{\partial}{\partial x_{j}}\left(A_{i} \boldsymbol{e}_{i}\right)=\frac{\partial A_{i}}{\partial x_{j}} \boldsymbol{e}_{j} \boldsymbol{e}_{i}=A_{i, j} \boldsymbol{e}_{j} \boldsymbol{e}_{i}$. The right gradient of a vector $\boldsymbol{A}$ is $\boldsymbol{A} \otimes \nabla \equiv\left(A_{i} \boldsymbol{e}_{i}\right) \otimes \boldsymbol{e}_{j} \frac{\partial}{\partial x_{j}}=\frac{\partial A_{i}}{\partial x_{j}} \boldsymbol{e}_{i} \otimes$ $\boldsymbol{e}_{j}=A_{i, j} \boldsymbol{e}_{i} \otimes \boldsymbol{e}_{j}$, or written as $\boldsymbol{A} \boldsymbol{\nabla} \equiv\left(A_{i} \boldsymbol{e}_{i}\right) \boldsymbol{e}_{j} \frac{\partial}{\partial x_{j}}=$ $\frac{\partial A_{i}}{\partial x_{j}} \boldsymbol{e}_{i} \boldsymbol{e}_{j}=A_{i, j} \boldsymbol{e}_{i} \boldsymbol{e}_{j}$, where $A_{i, j}=\frac{\partial A_{i}}{x_{j}}$.

The gradient of a scalar function is a vector, the divergence of a vector-valued function is a scalar $\boldsymbol{\nabla} \cdot \boldsymbol{A}$, and the gradient of a vector-valued function is a second-order tensor $\boldsymbol{\nabla} \boldsymbol{A}$. Although the del operator has some of the properties of a vector, it does not have them all, because it is an operator. For instance, $\boldsymbol{\nabla} \cdot \boldsymbol{A}$ is a scalar (called the divergence of $\boldsymbol{A}$ ) whereas $\boldsymbol{A} \cdot \boldsymbol{\nabla}$ is a scalar differential operator, where $\boldsymbol{A}$ is a vector. Thus the del operator $\boldsymbol{\nabla}$ does not commute in this sense.

It worth to emphasise that the right gradient of a vector $\boldsymbol{A} \boldsymbol{\nabla}$ is a more natural one, is often used in defining the deformation gradient tensor, displacement gradien$\mathrm{t}$ tensor, and velocity gradient tensor. It is clear that $\boldsymbol{A} \boldsymbol{\nabla}=(\boldsymbol{\nabla} \boldsymbol{A})^{T}$.

1. Mass density $\rho=\rho(\boldsymbol{x}, t)$ is a scalar-valued function of $\boldsymbol{x}$ and $t$ and its differential is $d \rho=\frac{\partial \rho}{\partial t} d t+\frac{\partial \rho}{\partial \boldsymbol{x}} \cdot \frac{\partial \boldsymbol{x}}{\partial t} d t$. For a scalar $\frac{\partial \rho}{\partial \boldsymbol{x}}=\rho \boldsymbol{\nabla}=\boldsymbol{\nabla} \rho$, so $d \rho=\frac{\partial \rho}{\partial t} d t+(\rho \boldsymbol{\nabla}) \cdot \boldsymbol{v} d t=$ $\frac{\partial \rho}{\partial t} d t+\boldsymbol{v} \cdot(\nabla \rho) d t$, or, dividing both sides by $d t$,

$$
\frac{d \rho}{d t}=\frac{\partial \rho}{\partial t}+(\rho \boldsymbol{\nabla}) \cdot \boldsymbol{v}=\frac{\partial \rho}{\partial t}+\boldsymbol{v} \cdot(\boldsymbol{\nabla} \rho) .
$$

2 . Flow velocity $\boldsymbol{v}=\boldsymbol{v}(\boldsymbol{x}, t)$ is a vector-valued function of $\boldsymbol{x}$ and $t$ and its differential is $d \boldsymbol{v}=\frac{\partial \boldsymbol{v}}{\partial t} d t+\frac{\partial \boldsymbol{v}}{\partial \boldsymbol{x}} \cdot \frac{\partial \boldsymbol{x}}{\partial t} d t$.
For a vector $\frac{\partial \boldsymbol{v}}{\partial \boldsymbol{x}}=\boldsymbol{v} \otimes \boldsymbol{\nabla}=\boldsymbol{v} \boldsymbol{\nabla}=(\boldsymbol{\nabla} \boldsymbol{v})^{T}$. Strictly speaking, the right gradient of $\boldsymbol{v}$ should be written as $\boldsymbol{v} \otimes \boldsymbol{\nabla} ;$ here, in order to agree as far as possible with conventional presentation, we write $\boldsymbol{v} \otimes \boldsymbol{\nabla}=\boldsymbol{v} \boldsymbol{\nabla}$ and, similarly for the left gradient, $\boldsymbol{\nabla} \otimes \boldsymbol{v}=\boldsymbol{\nabla} \boldsymbol{v}$. Hence $(\boldsymbol{v} \boldsymbol{\nabla})$. $\boldsymbol{v}=\boldsymbol{v} \cdot(\boldsymbol{\nabla} \boldsymbol{v})$ and $d \boldsymbol{v}=\frac{\partial \boldsymbol{v}}{\partial t} d t+(\boldsymbol{v} \boldsymbol{\nabla}) \cdot \boldsymbol{v} d t=\frac{\partial \rho}{\partial t} d t+\boldsymbol{v}$. $(\boldsymbol{\nabla} \boldsymbol{v}) d t$, or, dividing both sides by $d t$,

$$
\frac{d \boldsymbol{v}}{d t}=\frac{\partial \boldsymbol{v}}{\partial t}+(\boldsymbol{v} \boldsymbol{\nabla}) \cdot \boldsymbol{v}=\frac{\partial \boldsymbol{v}}{\partial t}+\boldsymbol{v} \cdot(\boldsymbol{\nabla} \boldsymbol{v})
$$

3. The 2 nd order stress tensor $\boldsymbol{\sigma}=\boldsymbol{\sigma}(\boldsymbol{x}, t)$ is a tensorvalued function of $\boldsymbol{x}$ and $t$ and its differential is $d \boldsymbol{\sigma}=$ $\frac{\partial \boldsymbol{\sigma}}{\partial t} d t+\frac{\partial \boldsymbol{\sigma}}{\partial \boldsymbol{x}} \cdot \frac{\partial \boldsymbol{x}}{\partial t} d t$. For an arbitrary tensor $\frac{\partial \boldsymbol{\sigma}}{\partial \boldsymbol{x}}=\boldsymbol{\sigma} \boldsymbol{\nabla} \neq$ $(\boldsymbol{\nabla} \boldsymbol{\sigma})^{T}$, hence $d \boldsymbol{\sigma}=\frac{\partial \boldsymbol{\sigma}}{\partial t} d t+(\boldsymbol{\sigma} \boldsymbol{\nabla}) \cdot \boldsymbol{v} d t$, or dividing both sides by $d t$ leads to $\frac{d \boldsymbol{\sigma}}{d t}=\frac{\partial \boldsymbol{\sigma}}{\partial t}+(\boldsymbol{\sigma} \boldsymbol{\nabla}) \cdot \boldsymbol{v}$. Since $(\boldsymbol{\sigma} \boldsymbol{\nabla}) \cdot \boldsymbol{v}=$ $\boldsymbol{v} \cdot(\nabla \sigma)$

$$
\frac{d \boldsymbol{\sigma}}{d t}=\frac{\partial \boldsymbol{\sigma}}{\partial t}+\boldsymbol{v} \cdot(\boldsymbol{\nabla} \boldsymbol{\sigma}) .
$$

If an operator must be introduced for the material derivative, it should be in the following form:

$$
\frac{d(:)}{d t}=\frac{\partial}{\partial t}(:)+\boldsymbol{v} \cdot[\boldsymbol{\nabla}(:)]
$$

For a scalar function $f(\boldsymbol{x}, t)$, this leads to $\frac{d f}{d t}=\frac{\partial f}{\partial t}+\boldsymbol{v}$. $(\nabla f)$. If $f$ is considered as a distributed function of gas molecules in their phase space, this derivative is as in Eq. (3.2) in Physical Kinetics by Lifshitz and Pitaevskii. [2]

For a vector function $\boldsymbol{u}(\boldsymbol{x}, t)$, Eq. (15) yields $\frac{d \boldsymbol{u}}{d t}=$ $\frac{\partial \boldsymbol{u}}{\partial t}+\boldsymbol{v} \cdot(\boldsymbol{\nabla u})$. For a tensor function $\boldsymbol{A}(\boldsymbol{x}, t)$, Eq. (15) yields $\frac{d \boldsymbol{A}}{d t}=\frac{\partial \boldsymbol{A}}{\partial t}+\boldsymbol{v} \cdot(\boldsymbol{\nabla} \boldsymbol{A})$.

The Navier-Stokes equations of incompressible flow can be expressed as follows: the momentum equation:

$$
\frac{\partial \boldsymbol{v}}{\partial t}+\boldsymbol{v} \cdot(\boldsymbol{\nabla} \boldsymbol{v})=-\frac{1}{\rho} \boldsymbol{\nabla} p+\nu \boldsymbol{\nabla}^{2} \boldsymbol{v}
$$

and mass conservation equation: $\boldsymbol{\nabla} \cdot \boldsymbol{v}=0$.

Applying the divergence operation to both sides of the momentum equation Eq.16 and use the mass conservation leads to a pressure equation: $\boldsymbol{\nabla}^{2} \cdot(p \mathbf{1})=$ $-\rho \boldsymbol{\nabla} \cdot(\boldsymbol{v} \cdot \boldsymbol{\nabla} \boldsymbol{v})=-\rho\left\{(\boldsymbol{\nabla} \boldsymbol{v})^{2}+[\boldsymbol{\nabla}(\boldsymbol{v} \boldsymbol{\nabla})] \cdot \boldsymbol{v}\right\}$. In which, $\boldsymbol{v}(\boldsymbol{x}, t)$ is flow velocity field, $\rho$ is constant mass density, $p(\boldsymbol{x}, t)$ is flow pressure, $\nu$ is kinematical viscosity, $t$ is time, $\boldsymbol{x}=x^{k} \boldsymbol{e}_{k}$ is position coordinates, $\boldsymbol{e}_{k}$ is a base vector and $\boldsymbol{v}$ is flow velocity, $\boldsymbol{\nabla}=\boldsymbol{e}_{k} \frac{\partial}{\partial x^{k}}$ is gradient operator and $\mathbf{1}=\boldsymbol{e}_{k} \boldsymbol{e}_{k}$ is an identity tensor, and $\boldsymbol{v} \boldsymbol{\nabla}=(\boldsymbol{\nabla} \boldsymbol{v})^{T}$.

Both solution existence and smoothness of the NavierStokes equations are still an open problem in mathematics [21], regardless of numerous abstract studies that have been done by mathematicians. Now the question is that, is it possible to propose an alternative and simple direct approach to express the flow velocity field without using complicated mathematics. Even we can not find the final solution of the velocity field, it is still plausible if we can get rid of the nonlinear convective term $\boldsymbol{v} \cdot(\boldsymbol{\nabla v})$. 
Assuming the determinant of the velocity gradient is not zero, namely det $\boldsymbol{\nabla} \boldsymbol{v} \neq 0$, the Navier-Stokes momentum equation in Eq.(16) can be rewritten as follows

$$
\boldsymbol{v}=\left[\boldsymbol{\nabla} \cdot\left(\nu \boldsymbol{\nabla} \boldsymbol{v}-\frac{p}{\rho} \mathbf{1}\right)-\frac{\partial \boldsymbol{v}}{\partial t}\right] \cdot(\boldsymbol{\nabla} \boldsymbol{v})^{-1}
$$

equivalently

$$
\boldsymbol{v}=(\boldsymbol{\nabla} \boldsymbol{v})^{-T} \cdot\left[\boldsymbol{\nabla} \cdot\left(\nu \boldsymbol{\nabla} \boldsymbol{v}-\frac{p}{\rho} \mathbf{1}\right)-\frac{\partial \boldsymbol{v}}{\partial t}\right] .
$$

The Eq.(17) and Eq.(18) can never be obtained, if the wrong material derivative $\frac{d}{d t}=\frac{\partial}{\partial t}+(\boldsymbol{v} \cdot \boldsymbol{\nabla})$ is used in the Navier-Stokes momentum equation as in the most of textbook such as [13].

Therefore we have a statement as follows:

$$
\begin{aligned}
& \text { The Navier-Stokes equation has solution if } \\
& \text { and only if the determinant of flow velocity } \\
& \text { gradient is not zero, namely } \operatorname{det}(\boldsymbol{\nabla} \boldsymbol{v}) \neq 0 \text {. }
\end{aligned}
$$

Although we have successfully split the velocity field $\boldsymbol{v}$ from the convective term $\boldsymbol{v} \cdot(\boldsymbol{\nabla} \boldsymbol{v})$, the calculation of the inverse of the velocity gradient $(\boldsymbol{\nabla v})^{-1}$ is still great challenge.

According to the Cayley-Hamilton theorem [22-24], for the 2nd order tensor $\boldsymbol{\nabla} \boldsymbol{v}$, the following polynomial holds:

$$
(\boldsymbol{\nabla} \boldsymbol{v})^{3}-I_{1}(\boldsymbol{\nabla} \boldsymbol{v})^{2}+I_{2} \boldsymbol{\nabla} \boldsymbol{v}-I_{3} \mathbf{1}=\mathbf{0},
$$

where $I_{1}=\operatorname{tr}(\boldsymbol{\nabla} \boldsymbol{v})=\boldsymbol{\nabla} \cdot \boldsymbol{v}, I_{2}=\frac{1}{2}\left[(\operatorname{tr} \boldsymbol{\nabla} \boldsymbol{v})^{2}-\operatorname{tr}(\boldsymbol{\nabla} \boldsymbol{v})^{2}\right]$ and $I_{3}=\operatorname{det}(\boldsymbol{\nabla} \boldsymbol{v})$. Hence, for the case of $\operatorname{det}(\boldsymbol{\nabla} \boldsymbol{v}) \neq 0$, we have:

$$
(\boldsymbol{\nabla} \boldsymbol{v})^{-1}=\frac{(\boldsymbol{\nabla} \boldsymbol{v})^{2}-I_{1} \boldsymbol{\nabla} \boldsymbol{v}+I_{2} \mathbf{1}}{\operatorname{det}(\boldsymbol{\nabla} \boldsymbol{v})}
$$

For incompressible flow, the divergence of velocity gradient is zero, namely, $I_{1}=\operatorname{tr}(\boldsymbol{\nabla} \boldsymbol{v})=\boldsymbol{\nabla} \cdot \boldsymbol{v}=0$, thus $I_{2}=\frac{1}{2}\left[(\operatorname{tr} \boldsymbol{\nabla} \boldsymbol{v})^{2}-\operatorname{tr}(\boldsymbol{\nabla} \boldsymbol{v})^{2}\right]=-\frac{1}{2} \operatorname{tr}(\boldsymbol{\nabla} \boldsymbol{v})^{2}$. Therefore, the inverse of the velocity gradient for incompressible flow takes a simpler form:

$$
(\boldsymbol{\nabla} \boldsymbol{v})^{-1}=\frac{(\boldsymbol{\nabla} \boldsymbol{v})^{2}-\frac{1}{2} \mathbf{1} t r(\boldsymbol{\nabla v})^{2}}{\operatorname{det}(\boldsymbol{\nabla} \boldsymbol{v})}
$$

Therefore, the incompressible flow velocity field in Eq.(17) is then reduced to the following form:

$$
\boldsymbol{v}=\frac{\left[\boldsymbol{\nabla} \cdot\left(\nu \boldsymbol{\nabla} \boldsymbol{v}-\frac{p}{\rho} \mathbf{1}\right)-\frac{\partial \boldsymbol{v}}{\partial t}\right] \cdot\left[(\boldsymbol{\nabla} \boldsymbol{v})^{2}-\frac{1}{2} \mathbf{1} \operatorname{tr}(\boldsymbol{\nabla} \boldsymbol{v})^{2}\right]}{\operatorname{det}(\boldsymbol{\nabla} \boldsymbol{v})}
$$

where $\boldsymbol{\nabla} \boldsymbol{v}=v_{j, i} \boldsymbol{e}_{i} \boldsymbol{e}_{j},(\boldsymbol{\nabla} \boldsymbol{v})^{2}=\boldsymbol{\nabla} \boldsymbol{v} \cdot \boldsymbol{\nabla} \boldsymbol{v}=v_{j, k} v_{k, i} \boldsymbol{e}_{i} \boldsymbol{e}_{j}$, $\operatorname{tr}(\boldsymbol{\nabla} \boldsymbol{v})^{2}=\mathbf{1}:(\boldsymbol{\nabla} \boldsymbol{v})^{2}=v_{i, k} v_{k, i}$ and $\operatorname{det}(\boldsymbol{\nabla} \boldsymbol{v})=$ $\varepsilon_{i j k} v_{1, i} v_{2, j} v_{3, k}$, and $\varepsilon_{i j k}$ is permutation symbol.

The great challenge to find solution of the NavierStokes equation are all from the existence of the velocity gradient $\boldsymbol{\nabla} \boldsymbol{v}$. Although the Eq.(17) and Eq.(22) can only give us a form-solution, but they still provide a rich information. Both Eq.(17) and Eq.(22) reveal that the current difficulty of finding a solution is because of the nonlinear term $\boldsymbol{v} \cdot(\boldsymbol{\nabla} \boldsymbol{v})$, or in other words, due to the existence of the velocity field gradient $\boldsymbol{\nabla} \boldsymbol{v}$. Accordingly, the solution of the Navier-Stokes equation will be blowup as $\operatorname{det} \boldsymbol{\nabla} \boldsymbol{v}$ tends to an infinitesimal, and has no solution when $\operatorname{det} \boldsymbol{\nabla} \boldsymbol{v}=0$.

The study might open an avenue for the understanding of turbulence. It would be important to check the value of $\operatorname{det} \boldsymbol{\nabla} \boldsymbol{v}$ for the study of flow stability and turbulence transition.

The mistake in the definition $\frac{d}{d t}=\frac{\partial}{\partial t}+(\boldsymbol{v} \cdot \boldsymbol{\nabla})$ is mainly the incorrect representation of $\boldsymbol{v} \cdot \boldsymbol{\nabla} ; \boldsymbol{\nabla}$ is a gradient operator rather than a vector, so that:

$$
\begin{aligned}
& \boldsymbol{v} \cdot \boldsymbol{\nabla}=\left(v_{1} \boldsymbol{e}_{1}+v_{2} \boldsymbol{e}_{2}+v_{3} \boldsymbol{e}_{3}\right) \cdot\left(\boldsymbol{e}_{1} \frac{\partial}{\partial x^{1}}+\boldsymbol{e}_{2} \frac{\partial}{\partial x^{2}}+\boldsymbol{e}_{3} \frac{\partial}{\partial x^{3}}\right) \\
& =\frac{\partial v_{1}}{\partial x^{1}}+\frac{\partial v_{2}}{\partial x^{2}}+\frac{\partial v_{3}}{\partial x^{3}} \neq v_{1} \frac{\partial}{\partial x^{1}}+v_{2} \frac{\partial}{\partial x^{2}}+v_{3} \frac{\partial}{\partial x^{3}} .
\end{aligned}
$$

In conclusion, the material derivative operator is correctly defined as: $\frac{d(:)}{d t}=\frac{\partial}{\partial t}(:)+\boldsymbol{v} \cdot[\nabla(:)]$. The solution existence condition of Navier-Stokes equation has been proposed from its form-solution, the conclusion is that "The Navier-Stokes equation has solution if and only if the determinant of flow velocity gradient is not zero, namely $\operatorname{det}(\boldsymbol{\nabla} \boldsymbol{v}) \neq 0 . "$

[1] L. D. Landau and E. M. Lifshitz, Fluid Mechanics (English version) (1987).

[2] E. M. Lifshitz and L. P. Pitaevskii, Physical Kinetics (Elsevier (Singapore) Ltd, 2008)

[3] L. Prandtl, Führer durch die Strömungslehre, Herbert Oertel (ed.) (Springer Vieweg, 2012).

[4] L. Prandtl, Prandtl's essentials of fluid mechanics, Herbert Oertel (ed.) (Springer-Verlag, 2004).

[5] J. D. Anderson, Computational Fluid Dynamics-the basics with applications (The McGraw-Hill Companies, Inc., 1995).

[6] S. B. Pope, Turbulent flows (Cambridge University Press, 2000).

[7] Y. A. Cengel and J. M. Cimbala, Fluid Mechanics: Fundamentals and Applications (McGraw-Hill Education, Inc., 2010).

[8] P. K. Kundu, I. M. Cohen and D. R. Dowling, Fluid Mechanics (Elsevier, 2012).

[9] G. Woan, The Cambridge Handbook of Physics Formulas, Cambridge University Press (2000)

[10] https://en.wikipedia.org/wiki/Gauge_covariant_derivative

[11] https://en.wikipedia.org/wiki/Navier_Stokes_equations

[12] https://www.britannica.com/science/Navier-Stokesequation

[13] L. D. Landau and E. M. Lifshitz, Fluid Mechanics (Russian version) (2017).

[14] J. Lighthill, Waves in Fluids (Cambridge University Press, 1978) 
[15] G. K. Batchelor, An introduction to fluid dynamics (Cambridge University Press, 2007)

[16] U. Frisch, Turbulence (Cambridge University Press, 1995)

[17] P.T. Nhan and M.D. Nam, Understanding viscoelasticity an introduction to rheology (Springer-Verlag Berlin Heidelberg,2013).

[18] X. C. Xie, Modern Tensor Analysis and Its Applications in Continuum Mechanics (Fudan University Press, 2014)

[19] Y. P. Zhao, Lectures on Mechanics (Science Press, China, 2018)
[20] J.N. Reddy, An Introduction to Continuum Mechanics, (Cambridge University Press NY, 2007).

[21] C.L. Fefferman, Existence and smoothness of the NavierStokes equation, The millennium prize problems, Clay Math. Institute, pp. 57-67(2006).

[22] W.R. Hamilton, Lectures on Quaternions. Dublin(1853).

[23] A. Cayley, A Memoir on the Theory of Matrices. Philos. Trans. 148.(1858).

[24] C. Truesdell, A first course in rational continuum mechanics. (Academic Press, New York, 1977). 